\title{
The Interaction Design of Outdoor Fitness Facilities Based on Solar Energy
}

\author{
Xiyin Gao, Yue Han and Ke Su \\ Agricultural University of Hebei, Baoding, Hebei, China
}

\begin{abstract}
At present, the functions of outdoor fitness facilities are single, and the modeling is stiff and bulky, so the user experience is very boring. The interaction design can not only enhance interaction between fitness facilities and persons, which add lively and interesting experience to users, but also successfully combine solar technology with fitness facilities, which makes the interaction design in public fitness facilities possible. In this article, fitness facilities consist of three parts: solar power modules, interactive modules, fitness module. Solar energy technology provides the interactive functions between users and products, such as interactive reaction, voice guidance, music player and the like. Considering the functions, modeling and interactivity, fitness facilities interaction design scheme based on solar energy is put into practice, which can improve appreciation and culture in public fitness facilities.
\end{abstract}

\section{Keywords-solar energy; fitness facilities; interaction design}

\section{INTRODUCTION}

With the rapid development of economy, persons have paid more and more attention to their health problems. As an important way to improve human health fitness exercise has become an indispensable part in life. The fitness facilities in community and other facilities whose roles are no longer a simple exercise apparatus but an important way to relieve stress and make body relax are the main instruments for ordinary people. And therefore when we design fitness facilities, we are not only conscious of the necessity to increase its potential functions and improve its security, but also take interactive design into consideration to give exercisers more care and communication. However, most public fitness facilities functions are single and its modeling is tedious. In order to make more interesting experience, exercisers often tend to take exercise together or carry music player with themselves. Using the environmentally friendly solar energy as a power supply module of interaction design in the fitness facilities is to establish some kind of interactive relationship between products and humans, which will put emotional elements into the design of fitness facilities and arouse user's some kind of emotion. So the existing fitness product image will be promoted, and users are willing to take fitness activities efficiently and pleasantly.

\section{A. Interaction Design of Outdoor Fitness Facilities}

From the investigation, we can find that the existing fitness facilities for the relationship between person and environment are relatively independent. On the one hand, there is no interaction between exercisers and fitness facilities, so exercise is monotonous and boring; on the other hand, the interaction between exercisers is only limited to language communication and therefore their fitness activities are independent. Fitness facilities product with simple modeling and rough manufacture doesn't own art forms and cultural connotation of beauty, so apparatus cannot be combined with the urban environment. It is difficult for apparatus to beautify the environment ${ }^{[1]}$. Most fitness persons feel boring when they are using fitness facilities. It urgently needs to improve interaction design of outdoor fitness facilities.

Interaction, put simply, is the process of continuous activities and reactions between two objects, no matter which is either biological or mechanical. The interaction design of outdoor fitness facilities emphasizes the following three aspects: on the one hand, the interaction between person and apparatus through adjustable or variable apparatus; on the other hand, the interpersonal interactions, namely by way of two or three exercisers together completing fitness movement; thirdly, the interaction between person and the environment. There is a certain relationship between apparatus and environment, which can bring exercisers emotional reaction or visual impact. Interaction design is introduced into the design of the fitness facilities, which not only can achieve the goal of effective fitness, but also meet the person's growing spiritual demand ${ }^{[2]}$.

\section{Prepare the Solar ENERgy Technology in the APPLICATION OF OUTDOOR FITNESS FACILITIES}

The technological breakthrough in single crystal cells and selective solar absorption coating is the landmark of the development of solar energy into contemporary ages, and is also the basis of the change of human energy utilization technology. Different ways of solar energy utilization decide its separate principle. Each has its characteristics and application scope ${ }^{[3]}$. Table 1 involves the comparison of the different ways of solar energy utilization performance, application range, etc. From it, we can safely draw the conclusion that the efficiency of solar energy acting as heat utilization can reach the lowest at about $30 \%$, up to $80 \%$; so solar technology is strongly feasible as power supply to serve for music player functions for outdoor fitness facilities.

Usually, outdoor fitness facilities are installed in the sunshine, which ensures the exercisers to enjoy the sunshine. So the combination of solar energy technology and apparatus has great possibilities. The outdoor interaction fitness system based on solar energy takes solar power to supply power for music player, video guide, motion information feedback, induction function in the fitness facilities. The significance of this innovation embodies in two points: firstly, using environmentally friendly solar power is green and no pollution so as to solve power supply problems in the process of outdoor 
fitness to transform from the traditional mechanical technology to intelligent information technology; secondly, the visualization and intelligent apparatus implements the outdoor fitness crowd motion real time data information collection and feedback.

TABLE I. THE PERFORMANCE AND APPLICATION OF SOLAR ENERGY UTILIZATION WAYS

\begin{tabular}{|c|c|c|c|c|c|c|}
\hline \multirow{8}{*}{ 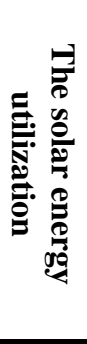 } & Solar energy utilization way & $\begin{array}{l}\text { The average } \\
\text { efficiency /\% }\end{array}$ & life span /a & $\begin{array}{l}\text { The initial } \\
\text { investment }\end{array}$ & $\begin{array}{c}\text { Technology } \\
\text { maturity }\end{array}$ & Environmentally friendly \\
\hline & illumination & $10 \sim 30$ & $20 \sim 30$ & high & high & high \\
\hline & water heater & $40 \sim 65$ & $5 \sim 15$ & medium & high & high \\
\hline & heating & $22 \sim 35$ & $15 \sim 20$ & medium & medium & high \\
\hline & refrigeration & $35 \sim 40$ & $10 \sim 15$ & high & medium & medium \\
\hline & Heat pump & $40 \sim 75$ & $10 \sim 15$ & medium & low & medium \\
\hline & Air collector & $50 \sim 80$ & $20 \sim 30$ & low & high & high \\
\hline & $\begin{array}{l}\text { Photovoltaic power } \\
\text { generation }\end{array}$ & $10 \sim 20$ & $10 \sim 15$ & high & high & low \\
\hline
\end{tabular}

Usually, outdoor fitness facilities are installed in the sunshine, which ensures the exercisers to enjoy the sunshine. So the combination of solar energy technology and apparatus has great possibilities. The outdoor interaction fitness system based on solar energy takes solar power to supply power for music player, video guide, motion information feedback, induction function in the fitness facilities. The significance of this innovation embodies in two points: firstly, using environmentally friendly solar power is green and no pollution so as to solve power supply problems in the process of outdoor fitness to transform from the traditional mechanical technology to intelligent information technology; secondly, the visualization and intelligent apparatus implements the outdoor fitness crowd motion real time data information collection and feedback.

\section{OUTDOOR INTERACTION FITNESS SYSTEM BASED ON SOLAR ENERGY}

The solar panel can supply power for a single body building apparatus or a plurality of fitness facilities. A number of fitness facilities and larger solar panels can compose a solar building where solar panels can be used as unit device to provide power and can also be used as an apparatus to block the sun. This thesis is the study of the solar energy combined with single apparatus.

\section{A. The Overall Design Scheme}

The outdoor interaction fitness facilities based on solar energy mainly include three parts: solar power supply module, interaction module and fitness module. These three systems work together to provide technical support for the establishment of human - machine interaction, user - user interaction, human - environment interaction, to ensure the exercise of fitness exercise for pleasant interaction.

1) Solar power supply module includes solar panels, inverters and batteries, mainly provides power for the interaction system. Through the solar cell board, electrical energy is stored in the battery, and then the power can be allocated to electrical apparatus.

2) Interaction module includes two parts: human machine interaction and interaction between human and environment. Human machine interaction includes infrared sensor and the control unit which is connected with the infrared sensor can control the start and stop of music player. Its main function is for the exercise to provide operational guidance, music player, information feedback, etc. The interaction of human and environment is achieved mainly from the fitness facilities modeling design.

3) Fitness module uses the existing apparatus and the newly developed fitness facilities, mainly to provide the function of physical exercise. In fitness module we can implement the interaction between persons, for example, two persons work together to complete a series of actions to achieve the object of the exercise, so we can design fitness facilities for parents and child or couples, etc.

\section{B. Interaction Module}

Interaction module is the main part of interaction design whose main role is to implement the interaction between exercisers and fitness facilities. When exercisers are ready for physical fitness activities, the infrared sensor installed in the interaction system can be induced to the person, and send signals to the control unit. At this point, the control unit opens music player to play voice, prompt exercisers information such as how to correct operation and play the music whose rhythm is the same as the fitness movement, and timely gives feedback to information such as time, exercise effect ${ }^{[4]}$. When the exercise is over and exercisers leave infrared sensors will feel the leave and send a signal to the control unit again, then control unit judges that no one uses the fitness facilities and then impel the music player to stop in order to save energy.

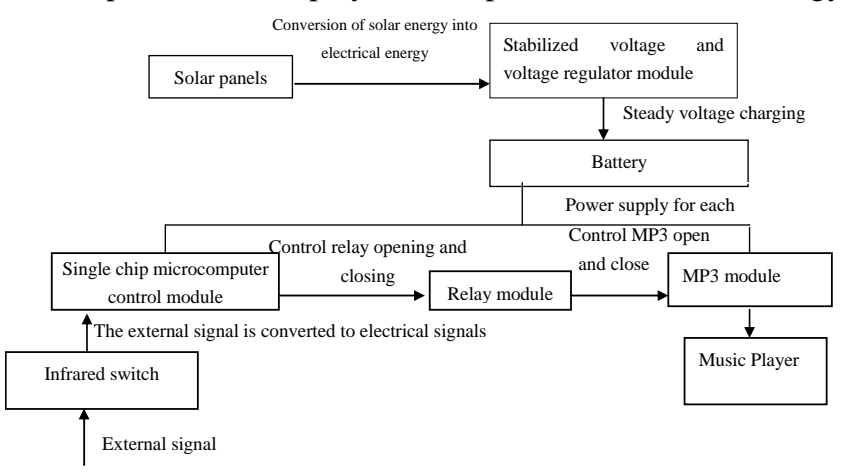

FIGURE I. SCHEMATIC DIAGRAM OF INTERACTION MODULE 


\section{The Structure of Interactive Outdoor Fitness Facilities Based on Solar Energy}

In order to ensure that the fitness facilities is convenient to be replaced, the solar panel is installed on a separate body, the model of the main body can be changed according to the needs of the design theme. Taking one design project for example, structure design is shown in Figure II.

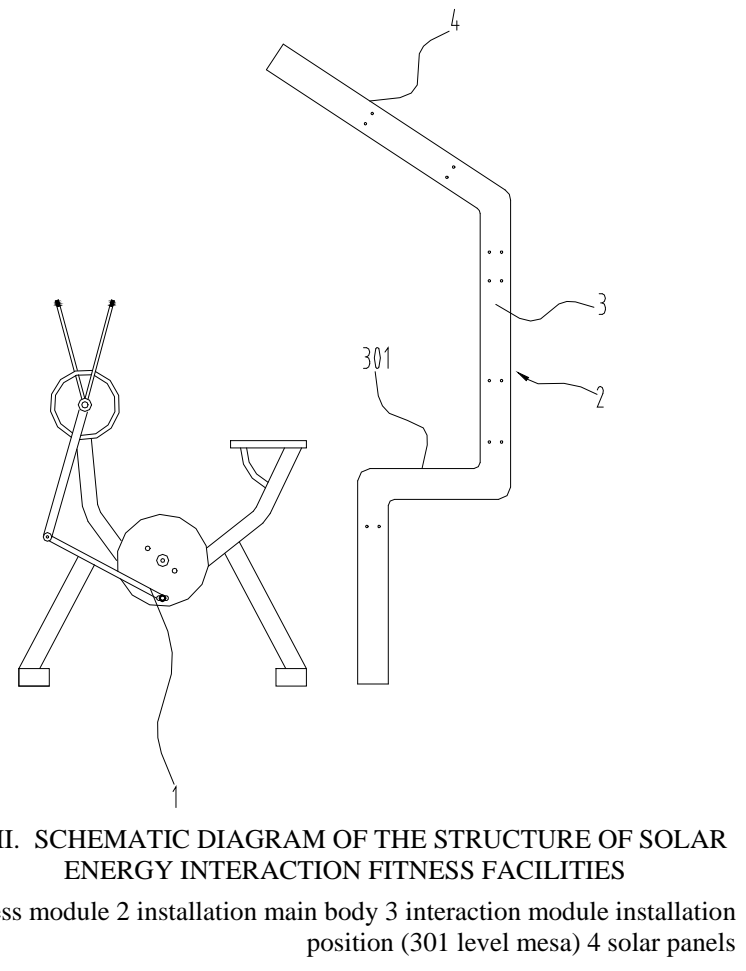

This design case for a stationary bike, whose fitness module can also be used for running machine or other apparatus just makes sure its position under the condition of induction in the interaction module. In addition, solar panels, such as sensors, music players and other components are installed in the position 3 shown in Figure II, to ensure the sensitivity of the sensor and voice play effect. Because the solar energy interaction fitness system is installed in the outdoor, material selection and structure design is to avoid the sun and man-made damage. Meanwhile, in order to ensure the full use of solar energy, the installation angle of the solar panel should be conducive to the transformation of solar energy.

\section{Landscape Type Solar Interaction Fitness Facilities Design}

In order to make the fitness facilities and the environment unified and coordinated, to perfect the urban functions and beautify the environment we put the solar energy interaction module independence, and integrate the local natural landscape or cultural environment factors into the modeling design, Figure III is one of independent solar interaction module which combines the pattern of ski movement with the streamline appearance. Around it, three different kinds of fitness facilities can be installed which can meet the different exercise needs. A landscape style solar interaction fitness facility in Figure IV combines the hula hoop movement curve, which improves the appreciation of the public sports facilities and culture. The fitness facilities are placed in the surrounding of the interaction module to beautify the exercise space. When person takes exercise, one not only can interact with the fitness facilities and meet exercise needs, but can also be immersed in the beautiful environment and meet the psychological needs.

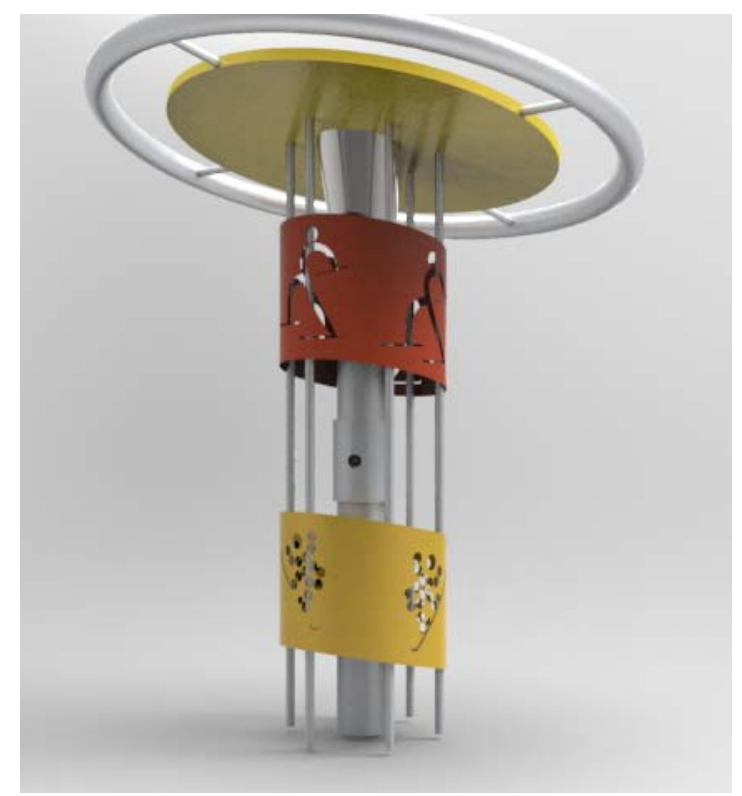

FIGURE III. INDEPENDENT SOLAR INTERACTION MODULE

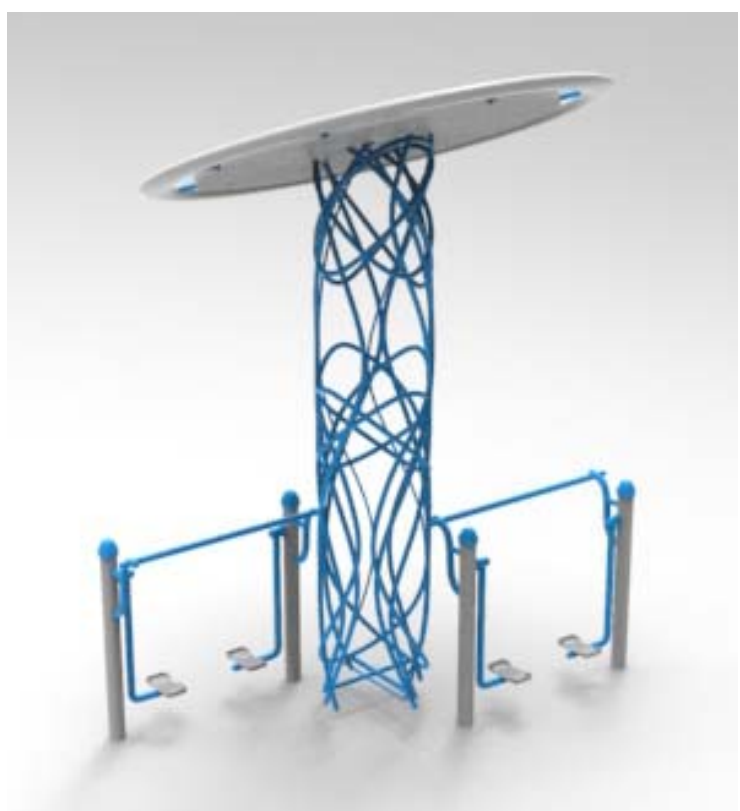

FIGURE IV. LANDSCAPE STYLE SOLAR INTERACTION FITNESS FACILITIES

\section{CONCLUSION}

The existing public fitness facilities in the market are single function, rough appearance and poor interaction. It is difficult to blend with the surrounding environment. This paper discusses the design of solar energy interaction fitness facilities 
by two design cases. The application of solar energy technology in the design ideas of outdoor fitness facilities promotes the interaction of product and person and spices up interesting experience, and improves the effect of fitness. The integration of culture into the fitness facilities modeling enhances the overall beauty between fitness facilities and environment. So far, one of the designs has been developed successfully. Through the experimental operation, solar energy technology is a good way to provide adequate power for interaction module of fitness facilities, which achieves fitness facilities interaction design. While satisfying exercise function, the interactive fitness facilities can meet the needs of person's emotional expression and beautify the environment ${ }^{[5]}$.

\section{ACKNOWLEDGEMENTS}

Fund project: Outdoor Health Industry Product Design and Development; Hebei Province Science and Technology Plan (no. : $15275702 \mathrm{~d}$ ).

\section{REFERENCES}

[1] [1] Li Dong, research on the design of public facilities in the urban environment, the master thesis of Northwest Agriculture and Forestry University, 2012.10.01:17

[2] [2] Zhang Xiaoling, Jing Shen good; "based on the new model of green fitness city sports park landscape construction of -- to Jinzhong sports park landscape design as an example"; Shanxi forestry science and technology in 20142 Vol. $43 \mathrm{Vol}$. 1

[3] [3] Bagnall MattBoreland., Photovoltaic Technolo-gies [J].Energypolicy [5]DarrenM, 2008, (36): 4390-4396

[4] [4]Yi Yanli, interaction design of outdoor fitness and recreation facilities, graduate thesis of Southwest Jiao Tong University, 2010

[5] Fund project: Outdoor Health Industry Product Design and Development; Hebei Province Science and Technology Plan (no. : 15275702 d). 